01,11

\title{
Размещение водорода в оксигидриде титана
}

\author{
(C) А.А. Валеева ${ }^{1,2}$, А.И. Гусев ${ }^{1}$ \\ ${ }^{1}$ Институт химии твердого тела УрО РАН, \\ Екатеринбург, Россия \\ ${ }^{2}$ Уральский федеральный университет им. Б.Н. Ельцина, \\ Екатеринбург, Россия \\ E-mail: gusev@ihim.uran.ru
}

Поступила в Редакцию 13 сентября 2021 г.

В окончательной редакции 13 сентября 2021 г.

Принята к публикации 26 сентября 2021 г.

\begin{abstract}
Рассмотрены возможные модели размещения атомов водорода на узлах кубической решетки оксигидрида титана $\mathrm{TiO}_{y} \mathrm{H}_{p}$ с вакансиями в металлической и неметаллической подрешетках. Установлено, что оксигидриды сохраняют кристаллическую решетку типа $B 1$ исходных кубических монооксидов и содержат структурные вакансии в металлической и кислородной подрешетках. Сопоставление полученных аналитических выражений для интенсивности дифракционных отражений с экспериментальными рентгеновскими и нейтронографическими данными показало, что в оксигидридах внедренные атомы Н занимают вакантные октаэдрические позиции $4(b)$ кислородной подрешетки, размещения Н в тетраэдрических позициях $8(c)$ не наблюдается. Найден канал перехода беспорядок-порядок, связанный с образованием упорядоченного моноклинного оксигидрида титана типа $\mathrm{Ti}_{5} \mathrm{O}_{5}$. Впервые рассчитаны функции распределения атомов $\mathrm{Ti}, \mathrm{O}$ и $\mathrm{H}$ в частично упорядоченном моноклинном оксигидриде $\mathrm{TiO}_{0.96} \mathrm{H}_{0.14}\left(\mathrm{Ti}_{0.89} \mathrm{O}_{0.85} \mathrm{H}_{0.12}\right)$ со структурой типа $\mathrm{Ti}_{5} \mathrm{O}_{5}$ и найдены концентрации указанных атомов на позициях его решетки.
\end{abstract}

Ключевые слова: монооксид титана, водород, нестехиометрия, вакансии, октаэдрические и тетраэдрические позиции, функция распределения.

DOI: $10.21883 /$ FTT.2022.01.51828.202

\section{1. Введение}

Нестехиометрический кубический монооксид титана $\mathrm{TiO}_{y} \equiv \mathrm{Ti}_{x} \mathrm{O}_{z} \quad\left(\mathrm{Ti}_{x} \mathbf{\square}_{1-x} \mathrm{O}_{z} \square_{1-z}\right.$, где $y=z / x, \square$ и $\mathbf{\square -}$ структурные вакансии неметаллической (кислородной) и металлической (титановой) подрешеток) со структурой типа $B 1$ принадлежит к группе сильно нестехиометрических соединений внедрения $M X_{y}(M=\mathrm{Ti}, \mathrm{Zr}, \mathrm{Hf}, \mathrm{V}$, $\mathrm{Nb}$, Ta $X=\mathrm{C}, \mathrm{N}, \mathrm{O})[1]$ и обладает широкой областью гомогенности от $\mathrm{TiO}_{0.80}$ до $\mathrm{TiO}_{1.25}$. В неупорядоченном состоянии атомы и структурные вакансии распределены по узлам соответствующих подрешеток случайным образом. Кубический монооксид титана с двойной дефектностью и его оксигидриды - перспективные материалы для водородной техники, фотокатализа, очистки воды от примесей, особенно в нанокристаллическом состоянии [2].

Нестехиометрические соединения $M X_{y}(X=\mathrm{C}, \mathrm{N}, \mathrm{O})$ со структурой типа $B 1$ могут поглощать водород из газовой фазы, образуя тройные гидридные фазы $M X_{y} \mathrm{H}_{p}$. Как правило, атомы водорода размещаются в вакантных октаэдрических позициях 4(b) неметаллической подрешетки. Например, в кубических карбидах титана и циркония атомы водорода занимают вакантные узлы углеродной подрешетки, т.е. октаэдрические междоузлия подрешетки металла [3-7]. Эксперименты по нейтронной дифракции $[5,8]$ показали, что гидрирование облегчает упорядочение углеродных вакансий в некоторых карбидах титана.
Нейтронография карбогидридов ниобия $[9,10]$ обнаружила, что в вакантных октаэдрических позициях атомы Н несколько смещены от центра в направлении $[100]_{B 1}$. Смещение атомов Н относительно центра вакансии обусловлено большим объемом вакансии, с одной стороны, и малым объемом атома водорода, с другой стороны.

Благодаря малому размеру атомы Н помимо вакантных октаэдрических позиций неметаллической подрешетки карбидов могут занимать тетраэдрические междоузлия. Возможное размещение атомов водорода в тетраэдрических междоузлиях следует из данных [11], согласно которым в некоторых кубических карбогидридах титана и ниобия $M \mathrm{C}_{y} \mathrm{H}_{p} \quad\left(\mathrm{TiC}_{0.64} \mathrm{H}_{0.38}\right.$, $\left.\mathrm{NbC}_{0.77} \mathrm{H}_{0.32}-\mathrm{NbC}_{1.00} \mathrm{H}_{0.25}\right)$ относительное содержание водорода, $p$, может превышать концентрацию структурных вакансий $(1-y)$ подрешетки углерода.

В литературе до последнего времени не было сведений о положении атомов Н в кубических оксигидридах титана $\mathrm{TiO}_{y} \mathrm{H}_{p}$. Обычно предполагается, что в оксигидридах $\mathrm{TiO}_{y} \mathrm{H}_{p}$ водород занимает вакантные узлы $4(b)$ кислородной подрешетки [12]. Однако размеры атома водорода таковы, что он может внедряться не только в октаэдрические, но и в тетраэдрические междоузлия гранецентрированной кубической (ГЦК) металлической решетки. Поэтому нельзя исключить, что хотя бы часть атомов водорода может размещаться в тетраэдрическихе междоузлиях гцк решетки титана. Действительно, в ку- 
бическом гидриде титана $\mathrm{TiH}_{y}$ с содержанием водорода $y \leq 1.8$ и структурой типа $C 1\left(\mathrm{CaF}_{2}\right)$ все атомы Н размещаются в тетраэдрических позициях $8(c)$ [13]. Таким образом, вопрос о том, в каких позициях кристаллической структуры оксигидрида титана размещаются атомы $\mathrm{H}$, до сих пор не ясен.

В связи с этим, цель настоящей работы - моделирование структуры кубических оксигидридов титана $\mathrm{TiO}_{y} \mathrm{H}_{p}$ и определение положения атомов $\mathrm{H}$ в них дифракционными методами.

\section{2. Результаты и обсуждение}

Модель структуры кубического (пр.гр. $F m \overline{3} m$ ) оксигидрида титана $\mathrm{TiO}_{y} \mathrm{H}_{p} \quad\left(\mathrm{Ti}_{x} \mathrm{O}_{z} \mathrm{H}_{p x}\right)$ с положением позиций $4(a), 4(b)$ и $8(c)$ показана на рис. 1. С учетом возможного внедрения водорода в окта- и тетрамеждоузлия кубической решетки, для кубического монооксида титана $\mathrm{Ti}_{x} \mathrm{O}_{z}$ можно предложить два варианта модельного размещения атомов Н. В первой модели, соответствующей кубической структуре типа $B 1$, водород может занимать только вакантные позиции 4(b) кислородной подрешетки. Во второй модели водород

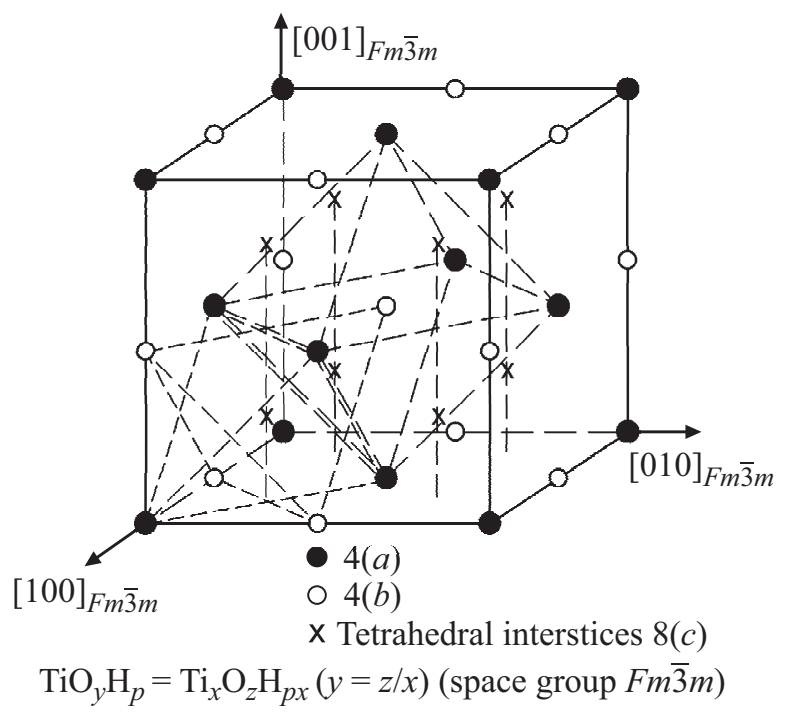

Рис. 1. Модель структуры кубического (пр. гр. $F m \overline{3} m$ ) оксигидрида титана $\mathrm{TiO}_{y} \mathrm{H}_{p}\left(\mathrm{Ti}_{x} \mathrm{O}_{z} \mathrm{H}_{p x}\right):(\bullet)$ позиции $4(a)-$ узлы металлической подрешетки, статистически занятые атомами Ті; (о) позиции 4(b) - узлы неметаллической подрешетки, статистически занятые атомами $\mathrm{O} ;(\times)$ позиции $8(c)-$ центры тетраэдрических междоузлий. Тетрамеждоузлия, образованные четырьмя узлами металлической подрешетки (или четырьмя узлами кислородной подрешетки), показаны коротким пунктиром. Если в оксигидриде $\mathrm{TiO}_{y} \mathrm{H}_{p}\left(\mathrm{Ti}_{x} \mathrm{O}_{z} \mathrm{H}_{p x}\right)$ атомы водорода размещаются только в вакантных позициях $4(b)$ кислородной подрешетки, то сохраняется структура типа $B 1$. Если в оксигидриде $\mathrm{TiO}_{y} \mathrm{H}_{p}$ часть атомов $\mathrm{H}$ занимает вакантные позиции $4(b)$ кислородной подрешетки, а остальные атомы $\mathrm{H}$ статистически размещаются в тетраэдрических позициях $8(c)$, то оксигидрид имеет структуру типа $D 0_{3}$. может занимать как вакантные позиции 4(b) кислородной подрешетки, так и часть тетрамеждоузлий, т. е. позиции $8(c)$. Эта модель соответствует кубической (пр. гр. $F m \overline{3} m$ ) структуре типа $D 0_{3}$. В этом случае формулу оксигидрида титана с учетом его структуры следует записывать как $\mathrm{TiO}_{y} \mathrm{H}_{p} \equiv \mathrm{Ti}_{x} \mathrm{O}_{z} \mathrm{H}_{p x} \equiv \mathrm{Ti}_{x} \mathrm{O}_{z} \mathrm{H}_{(p-q) x}^{4(b)}$ $\mathrm{H}_{q x}^{8(c)} \equiv \mathrm{Ti}_{x} \mathbf{\square}_{1-x} \mathrm{O}_{z} \mathrm{H}_{(p-q) x}^{4(b)} \square_{1-z-(p-q) x} \mathrm{H}_{q x}^{8(c)}$.

Обсудим, в каких кристаллографических позициях могут размещаться атомы водорода в оксигидридах титана и какие изменения могут наблюдаться на рентгенограммах и нейтронограммах оксигидридов титана при размещении атомов водорода только в позициях 4(b) или в позициях $4(b)$ и $8(c)$. Для этого найдем структурные амплитуды $F$ и структурные факторы $F^{2}$ монооксида титана $\mathrm{Ti}_{x} \mathrm{O}_{z} \equiv \mathrm{TiO}_{y}(y=z / x)$ со структурой $B 1$, оксигидрида титана $\mathrm{Ti}_{x} \mathrm{O}_{z} \mathrm{H}_{p x} \equiv \mathrm{TiO}_{y} \mathrm{H}_{p}$ со структурой $B 1$ и оксигидрида $\mathrm{Ti}_{x} \mathrm{O}_{z} \mathrm{H}_{p x} \equiv \mathrm{Ti}_{x} \mathrm{O}_{z} \mathrm{H}_{(p-q) x}^{4(b)} \mathrm{H}_{q x}^{8(c)}$ со структурой $D 0_{3}$. Структурная амплитуда

$$
F_{h k l}=\sum_{j} f_{j} \exp \left[-i 2 \pi\left(x_{j} h+y_{j} k+z_{j} l\right)\right]
$$

$\left(f_{j}\right.$ - атомный фактор рассеяния; $x_{j}, y_{j}, z_{j}-$ координаты $j$-го атома) определяет интенсивность дифракционных отражений. В общем случае структурная амплитуда является комплексной величиной, поэтому при анализе интенсивностей дифракционных отражений используется структурный фактор $F^{2}$, равный квадрату модуля структурной амплитуды (или произведению структурной амплитуды на комплексно-сопряженную величину $\left.F_{h k l}^{*}\right)$, т. е.

$$
F_{h k l}^{2}=\left|F_{h k l}\right|^{2}=F_{h k l} F_{h k l}^{*} .
$$

В кубической (пр.гр. $F m \overline{3} m)$ решетке со структурой $B 1$ позиции $4(a)$ имеют координаты (l 000$)$, $(1 / 21 / 20),(1 / 201 / 2),(01 / 21 / 2)$, а позиции 4(b) имеют координаты (1/2 1/2 1/2), ( $\left.\begin{array}{lll}0 & 0 & 1 / 2\end{array}\right),\left(\begin{array}{lll}0 & 1 / 2 & 0\end{array}\right)$ и (1/2 00 ). С учетом координат позиций $4(a)$ и 4(b), с вероятностями $x$ и $z$ статистически занятых атомами титана Ті и кислорода О, соответственно, структурная амплитуда $F$ кубического (пр. гр. $F m \overline{3} m)$ монооксида титана $\mathrm{Ti}_{x} \mathrm{O}_{z} \equiv \mathrm{TiO}_{y}(y=z / x)$ со структурой $B 1$ равна

$$
\begin{aligned}
F= & x f_{\mathrm{Ti}}\{1+\exp [-i \pi(h+k)]+\exp [-i \pi(h+l)] \\
& +\exp [-i \pi(k+l)]\}+z f_{\mathrm{O}}\{\exp [-i \pi(h+k+l)] \\
& +\exp (-i \pi h)+\exp (-i \pi k)+\exp (-i \pi l)]\},
\end{aligned}
$$

где $f_{\text {Ti и }} f_{\mathrm{O}}-$ факторы рассеяния излучения атомами Ті и О соответственно. В соответствии с $(1,2)$ структурный фактор $F^{2}$ для монооксида титана $\mathrm{Ti}_{x} \mathrm{O}_{z}$ со структурой $B 1$ имеет вид

$$
\begin{aligned}
F_{B 1}^{2}(h k l)= & \left\{x f_{\mathrm{Ti}}[1+\cos \pi(h+k)+\cos \pi(h+l)\right. \\
& +\cos \pi(k+l)]+z f_{\mathrm{O}}[\cos \pi(h+k+l) \\
& +\cos \pi h+\cos \pi k+\cos \pi l]\}^{2} .
\end{aligned}
$$


В оксигидриде титана $\mathrm{Ti}_{x} \mathrm{O}_{z} \mathrm{H}_{p x}$ со структурой $B 1$ позиции 4(a) статистически с вероятностью $x$ заняты атомами $\mathrm{Ti}$, а позиции $4(b)$ с вероятностями $z$ и $p x$ статистически заняты атомами $\mathrm{O}$ и $\mathrm{H}$, соответственно. С учетом этого структурная амплитуда $F$ кубического (пр.гр. $F m \overline{3} m$ ) оксигидрида титана $\mathrm{Ti}_{x} \mathrm{O}_{z} \mathrm{H}_{p x} \equiv \mathrm{TiO}_{y} \mathrm{H}_{p}$ со структурой В1 имеет вид

$$
\begin{aligned}
& F=x f_{\mathrm{Ti}}\{1+\exp [-i \pi(h+k)]+\exp [-i \pi(h+l)] \\
& +\exp [-i \pi(k+l)]\}+\left(z f_{\mathrm{O}}+p x f_{\mathrm{H}}\right)\{\exp [-i \pi(h+k+l)] \\
& +\exp (-i \pi h)+\exp (-i \pi k)+\exp (-i \pi l)]\}
\end{aligned}
$$

где $f_{\mathrm{H}}-$ фактор рассеяния излучения атомами водорода Н. Структурный фактор $F^{2}$ равен

$$
\begin{aligned}
& F_{B 1}^{2}(h k l)=\left\{x f_{\mathrm{Ti}}[1+\cos \pi(h+k)+\cos \pi(h+l)\right. \\
& \quad+\cos \pi(k+l)]+\left(z f_{\mathrm{O}}+p x f_{\mathrm{H}}\right)[\cos \pi(h+k+l) \\
& \quad+\cos \pi h+\cos \pi k+\cos \pi l]\}^{2} .
\end{aligned}
$$

Если в оксигидрида $\mathrm{Ti}_{x} \mathrm{O}_{z} \mathrm{H}_{p x}$ атомы водорода могут размещаться как в незаполненных узлах кислородной подрешетки (позициях $4(b))$, так и в тетрамеждоузлиях (позициях $8(c)$ ), то оксигидрид титана имеет кубическую (пр.гр. $F m \overline{3} m)$ структуру типа $D_{3}$ и его формулу можно записать как $\mathrm{Ti}_{x} \mathrm{O}_{z} \mathrm{H}_{p x} \equiv \mathrm{Ti}_{x} \mathrm{O}_{z} \mathrm{H}_{(p-q) x}^{4(b)} \mathrm{H}_{q x}^{8(c)}$. В таком оксигидриде титана позиции $4(a)$ статистически с вероятностью х заняты атомами Тi, позиции $4(b)$ статистически с вероятностями $z$ и $(p-q) x$ заняты атомами кислорода $\mathrm{O}$ и водорода $\mathrm{H}$, соответственно, и позиции $8(c)$ статистически с вероятностью $q x / 2$ заняты атомами Н. Позиции $8(c)$ кубической (пр.гр. $F m \overline{3} m$ ) решетки имеют координаты: $(1 / 41 / 41 / 4),(3 / 43 / 41 / 4)$, $(3 / 41 / 43 / 4),(1 / 43 / 43 / 4),(3 / 43 / 43 / 4),(1 / 41 / 43 / 4)$, $(1 / 43 / 41 / 4)$ и $(3 / 41 / 41 / 4)$. С учетом координат позиций $4(a), 4(b)$ и $8(c)$ и вероятностей их заполнения атомами $\mathrm{Ti}$, О и $\mathrm{H}$ структурная амплитуда $F$ неупорядоченного кубического (пр.гр. $F m \overline{3} m$ ) оксигидрида $\mathrm{Ti}_{x} \mathrm{O}_{z} \mathrm{H}_{(p-q) x}^{4(b)} \mathrm{H}_{q x}^{8(c)}$ со структурой $D 0_{3}$ имеет следующий вид

$$
\begin{aligned}
& F=x\left[f_{\mathrm{Ti}}\{1+\exp [-i \pi(h+k)]+\exp [-i \pi(h+l)]\right. \\
& +\exp [-i \pi(k+l)]\}+\left(y f_{\mathrm{O}}+(p-q) f_{\mathrm{H}}\right) \\
& \times\{\exp [-i \pi(h+k+l)]+\exp (-i \pi h)+\exp (-i \pi k) \\
& +\exp (-i \pi l)]\}+\left(q f_{\mathrm{H}} / 2\right)\{\exp [-i \pi(h+k+l) / 2] \\
& +\exp [-i \pi(3 h+3 k+l) / 2]+\exp [-i \pi(3 h+k+3 l) / 2] \\
& +\exp [-i \pi(h+3 k+3 l) / 2]+\exp [-i \pi(3 h+3 k+3 l) / 2] \\
& +\exp [-i \pi(h+k+3 l) / 2]+\exp [-i \pi(h+3 k+l) / 2] \\
& +\exp [-i \pi(3 h+k+l) / 2]\} .
\end{aligned}
$$

В соответствии с (6) структурный фактор $F^{2}$ неупорядоченного кубического (пр.гр. $F m \overline{3} m$ ) оксигидрида титана $\mathrm{Ti}_{x} \mathrm{O}_{z} \mathrm{H}_{(p-q) x}^{4(b)} \mathrm{H}_{q x}^{8(c)}$ со структурой типа $D 0_{3}$ равен

$$
\begin{aligned}
& F_{D \mathrm{O}_{3}}^{2}(h k l)=x^{2}\left\{f_{\mathrm{Ti}}[1+\cos \pi(h+k)+\cos \pi(h+l)\right. \\
& \quad+\cos \pi(k+l)]+\left[y f_{\mathrm{O}}+(p-q) f_{\mathrm{H}}\right][\cos \pi(h+k+l) \\
& \quad+\cos \pi h+\cos \pi k+\cos \pi l]+\frac{q}{2} f_{\mathrm{H}}\left[\cos \frac{\pi}{2}(h+k+l)\right. \\
& \quad+\cos \frac{\pi}{2}(3 h+3 k+l)+\cos \frac{\pi}{2}(3 h+k+3 l) \\
& \quad+\cos \frac{\pi}{2}(h+3 k+3 l)+\cos \frac{\pi}{2}(3 h+3 k+3 l) \\
& \quad+\cos \frac{\pi}{2}(h+k+3 l)+\cos \frac{\pi}{2}(h+3 k+l) \\
& \left.\left.\quad+\cos \frac{\pi}{2}(3 h+k+l)\right]\right\}^{2}+x^{2}\left\{\frac { q } { 2 } f _ { \mathrm { H } } \left[\sin \frac{\pi}{2}(h+k+l)\right.\right. \\
& \quad+\sin \frac{\pi}{2}(3 h+3 k+l)+\sin \frac{\pi}{2}(3 h+k+3 l) \\
& \quad+\sin \frac{\pi}{2}(h+3 k+3 l)+\sin \frac{\pi}{2}(3 h+3 k+3 l) \\
& \quad+\sin \frac{\pi}{2}(h+k+3 l)+\sin \frac{\pi}{2}(h+3 k+3 l) \\
& \left.\left.+\sin \frac{\pi}{2}(3 h+k+l)\right]\right\}^{2} .
\end{aligned}
$$

Из формул (5) и (7) ясно, что заполнение позиций 8(c) атомами Н не приводит к появлению новых дифракционных отражений, а лишь незначительно меняет величину структурных факторов (табл. 1). Поэтому неупорядоченные кубические оксигидриды $\mathrm{Ti}_{x} \mathrm{O}_{z} \mathrm{H}_{p x}$ со структурами $B 1$ и $D 0_{3}$ имеют одинаковый набор дифракционных отражений. Поскольку фактор рассеяния рентгеновского излучения атомами $\mathrm{H}$ очень мал по сравнению с факторами рассеяния атомами Ті и $\mathrm{O}$, то внедрение водорода слабо сказывается на интенсивности рентгеновских отражений. Однако рентгеновская дифракция позволяет выявить упорядочение атомов Ті и $\mathrm{O}$ в монооксиде или оксигидриде титана.

В случае нейтронографических измерений ситуация совершенно иная. Амплитуды когерентного рассеяния нейтронов ядрами $\mathrm{Ti},{ }^{16} \mathrm{O}$ и ${ }^{1} \mathrm{H}$ равны $-3.438 \cdot 10^{-15}$, $5.803 \cdot 10^{-15}$ и $-3.741 \cdot 10^{-15} \mathrm{~m}$, соответственно $[14,15]$. Амплитуды рассеяния нейтронов $f_{\text {Ti }}$ и $f_{\mathrm{O}}$ противоположны по знаку, поэтому даже в монооксиде титана структурные факторы четных отражений очень малы по сравнению со структурными факторами нечетных отражений. Благодаря отрицательной амплитуде рассеяния нейтронов ядрами ${ }^{1} \mathrm{H}$ внедрение водорода в монооксид титана приводит к еще большему относительному уменьшению структурных факторов $F^{2}$ четных отражений (200), (220), (222), (400), (420) и (422) по 
Таблица 1. Структурные факторы $F^{2}$ кубических (пр. гр. $F m \overline{3} m$ ) монооксида титана $\mathrm{Ti}_{x} \mathrm{O}_{z} \equiv \mathrm{TiO}_{y}$ (структура $B 1$ ) и оксигидридов титана $\mathrm{Ti}_{x} \mathrm{O}_{z} \mathrm{H}_{p x} \equiv \mathrm{TiO}_{y} \mathrm{H}_{p}$ (структуры $D 0_{3}$ и $\left.B 1\right)(P-$ фактор повторяемости)

\begin{tabular}{c|r|c|c|c}
\hline \multirow{2}{*}{$(h k l)$} & \multirow{2}{*}{$P$} & \multicolumn{3}{|c}{$F^{2}$} \\
\cline { 3 - 5 } & & $\mathrm{Ti}_{x} \mathrm{O}_{z} \equiv \mathrm{TiO}_{y}(y=z / x), B 1$ & $\mathrm{Ti}_{x} \mathrm{O}_{z} \mathrm{H}_{p x} \equiv \mathrm{Ti}_{x} \mathrm{O}_{z} \mathrm{H}_{(p-q) x}^{(4(b))} \mathrm{H}_{q x}^{8(c)}, D 0_{3}$ & $\operatorname{Ti}_{x} \mathrm{O}_{z} \mathrm{H}_{p x} \equiv \mathrm{TiO}_{y} \mathrm{H}_{p}(y=z / x), B 1$ \\
\hline 111 & 8 & $16 x^{2}\left(f_{\mathrm{Ti}}-y f_{\mathrm{O}}\right)^{2}$ & $16 x^{2}\left\{f_{\mathrm{Ti}}-\left[y f_{\mathrm{O}}+(p-q) f_{\mathrm{H}}\right]\right\}^{2}$ & $16 x^{2}\left[f_{\mathrm{Ti}}-\left(y f_{\mathrm{O}}+p f_{\mathrm{H}}\right)\right]^{2}$ \\
200 & 6 & $16 x^{2}\left(f_{\mathrm{Ti}}+y f_{\mathrm{O}}\right)^{2}$ & $16 x^{2}\left[f_{\mathrm{Ti}}+y f_{\mathrm{O}}+(p-2 q) f_{\mathrm{H}}\right]^{2}$ & $16 x^{2}\left(f_{\mathrm{Ti}}+y f_{\mathrm{O}}+p f_{\mathrm{H}}\right)^{2}$ \\
220 & 12 & $16 x^{2}\left(f_{\mathrm{Ti}}+y f_{\mathrm{O}}\right)^{2}$ & $16 x^{2}\left(f_{\mathrm{Ti}}+y f_{\mathrm{O}}+p f_{\mathrm{H}}\right)^{2}$ & $16 x^{2}\left(f_{\mathrm{Ti}}+y f_{\mathrm{O}}+p f_{\mathrm{H}}\right)^{2}$ \\
311 & 24 & $16 x^{2}\left(f_{\mathrm{Ti}}-y f_{\mathrm{O}}\right)^{2}$ & $16 x^{2}\left\{f_{\mathrm{Ti}}-\left[y f_{\mathrm{O}}+(p-q) f_{\mathrm{H}}\right]\right\}^{2}$ & $16 x^{2}\left[f_{\mathrm{Ti}}-\left(y f_{\mathrm{O}}+p f_{\mathrm{H}}\right)\right]^{2}$ \\
222 & 8 & $16 x^{2}\left(f_{\mathrm{Ti}}+y f_{\mathrm{O}}\right)^{2}$ & $16 x^{2}\left[f_{\mathrm{Ti}}+y f_{\mathrm{O}}+(p-2 q) f_{\mathrm{H}}\right]^{2}$ & $16 x^{2}\left(f_{\mathrm{Ti}}+y f_{\mathrm{O}}+p f_{\mathrm{H}}\right)^{2}$ \\
400 & 6 & $16 x^{2}\left(f_{\mathrm{Ti}}+y f_{\mathrm{O}}\right)^{2}$ & $16 x^{2}\left(f_{\mathrm{Ti}}+y f_{\mathrm{O}}+p f_{\mathrm{H}}\right)^{2}$ & $16 x^{2}\left(f_{\mathrm{Ti}}+y f_{\mathrm{O}}+p f_{\mathrm{H}}\right)^{2}$ \\
331 & 24 & $16 x^{2}\left(f_{\mathrm{Ti}}-y f_{\mathrm{O}}\right)^{2}$ & $16 x^{2} f_{\mathrm{Ti}}-\left[y f_{\mathrm{O}}+(p-q) f_{\mathrm{H}}\right]^{2}$ & $16 x^{2}\left[f_{\mathrm{Ti}}-\left(y f_{\mathrm{O}}+p f_{\mathrm{H}}\right)\right]^{2}$ \\
420 & 24 & $16 x^{2}\left(f_{\mathrm{Ti}}+y f_{\mathrm{O}}\right)^{2}$ & $16 x^{2}\left[f_{\mathrm{Ti}}+y f_{\mathrm{O}}+(p-2 q) f_{\mathrm{H}}\right]^{2}$ & $16 x^{2}\left(f_{\mathrm{Ti}}+y f_{\mathrm{O}}+p f_{\mathrm{H}}\right)^{2}$ \\
422 & 24 & $16 x^{2}\left(f_{\mathrm{Ti}}+y f_{\mathrm{O}}\right)^{2}$ & $16 x^{2}\left(f_{\mathrm{Ti}}+y f_{\mathrm{O}}+p f_{\mathrm{H}}\right)^{2}$ & $16 x^{2}\left(f_{\mathrm{Ti}}+y f_{\mathrm{O}}+p f_{\mathrm{H}}\right)^{2}$ \\
333 & 8 & $16 x^{2}\left(f_{\mathrm{Ti}}-y f_{\mathrm{O}}\right)^{2}$ & $\left.16 x^{2}\left\{f_{\mathrm{Ti}}-\left[y f_{\mathrm{O}}+(p-q) f_{\mathrm{H}}\right)\right]\right\}^{2}$ & $16 x^{2}\left[f_{\mathrm{Ti}}-\left(y f_{\mathrm{O}}+p f_{\mathrm{H}}\right)\right]^{2}$ \\
511 & 24 & $16 x^{2}\left(f_{\mathrm{Ti}}-y f_{\mathrm{O}}\right)^{2}$ & $\left.16 x^{2}\left\{f_{\mathrm{Ti}}-\left[y f_{\mathrm{O}}+(p-q) f_{\mathrm{H}}\right)\right]\right\}^{2}$ & $16 x^{2}\left[f_{\mathrm{Ti}}-\left(y f_{\mathrm{O}}+p f_{\mathrm{H}}\right)\right]^{2}$
\end{tabular}

сравнению с факторами $F^{2}$ нечетных отражений (111), (311), (331) и (511). При внедрении водорода только в вакантные позиции $4(b)$, т.е. в оксигидриде $\mathrm{TiO}_{y} \mathrm{H}_{p}$ $\left(\mathrm{Ti}_{x} \mathrm{O}_{z} \mathrm{H}_{p x}\right)$ структурные факторы $F^{2}$ (без учета углового ослабления интенсивности) всех четных отражений равны $16 x^{2}\left(f_{\mathrm{Ti}}+y f_{\mathrm{O}}+p f_{\mathrm{H}}\right)^{2}$ (табл. 1). Если водород размещается также в позициях $8(c)$ и оксигидрид $\mathrm{Ti}_{x} \mathrm{O}_{x} \mathrm{H}_{(p-q) x}^{4(b)} \mathrm{H}_{q x}^{8(c)}$ имеет структуру типа $D 0_{3}$, то структурные факторы $F^{2}=16 x^{2}\left(f_{\mathrm{Ti}}+y f_{\mathrm{O}}+p f_{\mathrm{H}}\right)^{2}$ четных отражений $(h k l) \mathrm{c}(h+k+l)=4 n$ (где $n-$ целое число), оказываются меньше структурных факторов $F^{2}=16 x^{2}\left[f_{\mathrm{Ti}}+y f_{\mathrm{O}}+(p-2 q) f_{\mathrm{H}}\right]^{2}$ остальных четных отражений. Поэтому уточнение структуры путем анализа соотношения интенсивностей нейтронографических дифракционных отражений позволяет определить, какие позиции занимает водород в оксигидриде титана.

Для выяснения того, какие позиции занимают атомы водорода в оксигидридах титана, были получены закаленные образцы неупорядоченного монооксида титана $\mathrm{TiO}_{0.72} \quad\left(\mathrm{Ti}_{0.95} \square_{0.05} \mathrm{O}_{0.69} \square_{0.31}\right)$ и $\mathrm{TiO}_{0.96}$ $\left(\mathrm{Ti}_{0.89} \square_{0.11} \mathrm{O}_{0.85} \square_{0.15}\right)$ со структурными вакансиями как в подрешетке титана, так и в подрешетке кислорода. Подробно условия синтеза монооксида титана в неупорядоченном и упорядоченном состояниях описаны ранее $[16,17]$. Оба закаленных образца содержали только неупорядоченную кубическую (пр. гр. $F m \overline{3} m$ ) фазу $\mathrm{TiO}_{y}$ co структурой типа $B 1$, период $a_{B 1}$ кристаллической решетки неупорядоченных монооксидов $\mathrm{TiO}_{0.72}$ и $\mathrm{TiO}_{0.96}$ равен 0.41934 и $0.41827 \mathrm{~nm}$ соответственно. По составу образец $\mathrm{TiO}_{0.96}$ близок к эквиатомному монооксиду $\mathrm{TiO}_{1.00}$, в котором при температуре ниже $1250-1300 \mathrm{~K}$ образуется моноклинная (пр.гр. $C 2 / m$ ) упорядоченная фаза $\mathrm{Ti}_{5} \mathrm{O}_{5}\left(\mathrm{Ti}_{5} \square \mathrm{O}_{5} \square\right)[18]$.

Гидрирование закаленных неупорядоченных образцов кубического монооксида титана $\mathrm{TiO}_{y}$ проводили в вакуумной системе типа Сивертса в водороде $\mathrm{H}_{2}$ при атмосферном давлении и температуре $973 \mathrm{~K}$.
Кристаллическую структуру образцов монооксида титана $\mathrm{TiO}_{y} \equiv \mathrm{Ti}_{x} \mathrm{O}_{z}$ и гидрированных образцов $\mathrm{TiO}_{y} \mathrm{H}_{p} \equiv \mathrm{Ti}_{x} \mathrm{O}_{z} \mathrm{H}_{p x}$ изучали на рентгеновском автодифрактометре Shimadzu XRD-7000 методом Брегга-Брентано в $\mathrm{Cu} K \alpha_{1,2}$-излучении в интервале углов $2 \theta$ от 10 до $157^{\circ}$ с шагом $\Delta(2 \theta)=0.02^{\circ}$ и экспозицией $10 \mathrm{~s}$ в каждой точке. Распределение атомов водорода $\mathrm{H}$ в кристаллической решетке оксигидридов титана $\mathrm{TiO}_{y} \mathrm{H}_{p}$ исследовали методом структурной нейтронографии на порошковом дифрактометре высокого разрешения ВТ1 [19] в центре нейтронных исследований NIST (Gaithersburg, MD, USA). Длина волны нейтронов составляла $0.15401 \mathrm{~nm}$, нейтронограммы записывали в интервале углов $5 \leq 2 \theta \leq 162^{\circ}$ с шагом $\Delta(2 \theta)=0.05^{\circ}$.

Определение фазового состава образцов и параметров кристаллической решетки фаз, а также окончательное уточнение структуры образцов проводили с помощью программного пакета X'Pert Plus [20]. Профиль дифракционных отражений моделировали функцией псевдо-Фойгта, фон описывали полиномом Чебышева 5-го порядка.

В результате гидрирования неупорядоченного монооксида титана $\mathrm{TiO}_{0.72}$ образовался кубический (пр.гр. $F m \overline{3} m$ ) оксигидрид $\mathrm{TiO}_{0.72} \mathrm{H}_{0.30}$ с периодом кристаллической решетки $0.42076 \mathrm{~nm}$ (рис. 2) [21]. Увеличение периода решетки от 0.41934 до $0.42076 \mathrm{~nm}-$ следствие внедрения большого количества водорода в кристаллическую решетку монооксида $\mathrm{TiO}_{y}$.

Уточнение структуры однофазного оксигидрида $\mathrm{TiO}_{0.72} \mathrm{H}_{0.30}$ с помощью программы X'Pert Plus [20] и с учетом структурных факторов $F^{2}$ (см. табл. 1) дало следующие результаты. При описании нейтронограммы (рис. 2,b) в модели со структурой $B 1$ факторы сходимости Ритвелда равны $R_{\text {exp }}=0.067, R_{p}=0.055$, $R_{\text {wp }}=0.072 \quad$ и $\quad R_{\mathrm{I}}\left(R_{\text {Bragg }}\right)=0.015 . \quad$ Использование модели структуры типа $D 0_{3}$ с размещением четверти 
всех атомов водорода в тетраэдрических позициях (в этом случае степень заполнения тетрапозиций 8(c) атомами $\mathrm{H}$ равна 0.036) привело к ухудшению сходимости: $R_{\exp }=0.069, R_{\mathrm{p}}=0.056, R_{\mathrm{wp}}=0.073$ и $R_{\mathrm{I}}\left(R_{\text {Bragg }}\right)=0.018$. Увеличение степени заполнения позиций $8(c)$ атомами Н от 0.036 до 0.144 , т. е. размещение всех атомов водорода в тетраэдрических позициях 8(c) еще больше ухудшило сходимость экспериментальных и рассчитанных дифрактограмм. Таким образом, уточнение структуры оксигидрида $\mathrm{TiO}_{0.72} \mathrm{H}_{0.30}$ с использованием рентгеновских и нейтронографических данных показало, что этот оксигидрид имеет кубическую (пр. гр. $F m \overline{3} m)$ структуру типа $B 1$, в которой все атомы водорода находятся в позициях $4(b)$. С учетом степеней заполнения позиций 4(a) атомами Ті и позиций 4(b) атомами О и Н оксигидрид имеет состав $\mathrm{Ti}_{0.96} \mathrm{O}_{0.69} \mathrm{H}_{0.29}$.
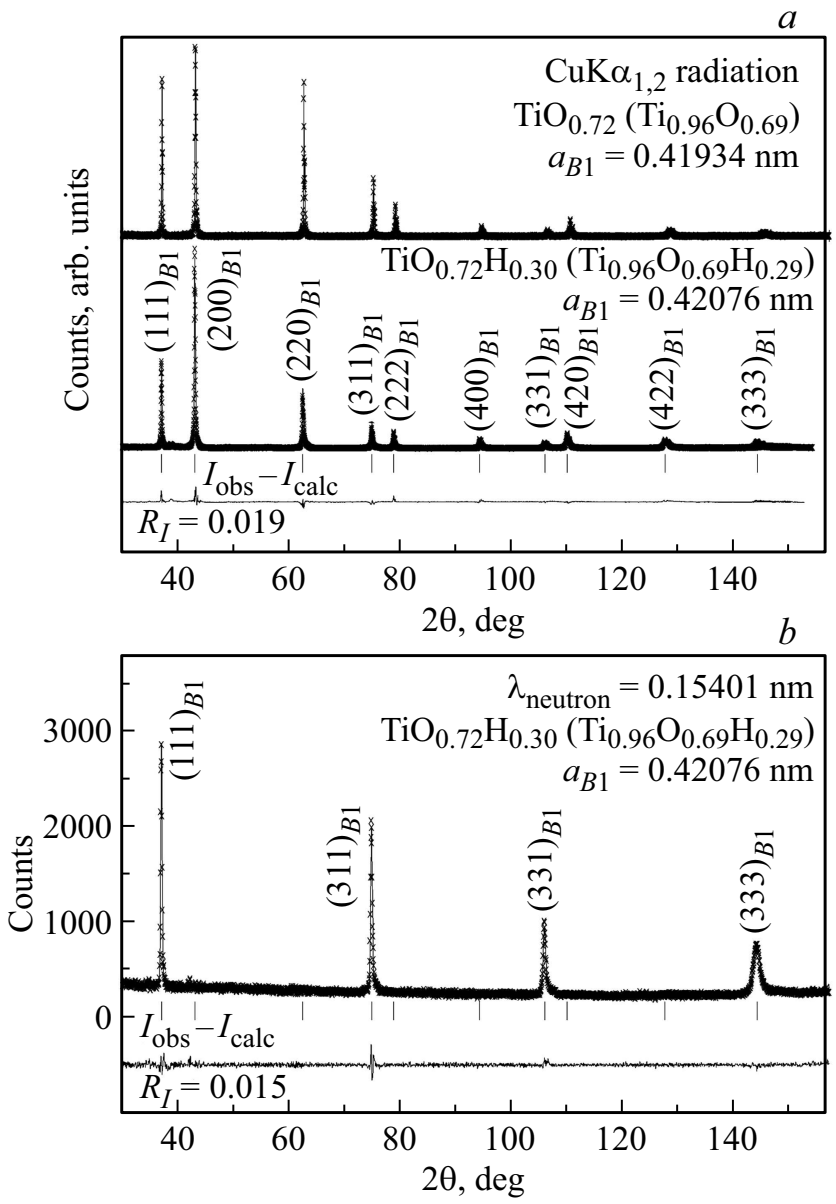

Рис. 2. Рентгенограммы $(a)$ исходного неупорядоченного кубического монооксида титана $\mathrm{TiO}_{0.72}\left(\mathrm{Ti}_{0.96} \mathrm{O}_{0.69}\right)$ и оксигидрида титана $\mathrm{TiO}_{0.72} \mathrm{H}_{0.30}\left(\mathrm{Ti}_{0.96} \mathrm{O}_{0.69} \mathrm{H}_{0.29}\right)$ и нейтронограмма $(b)$ оксигидрида титана $\mathrm{TiO}_{0.72} \mathrm{H}_{0.30}\left(\mathrm{Ti}_{0.96} \mathrm{O}_{0.69} \mathrm{H}_{0.29}\right)(\times-$ эксперимент, сплошная линия - расчет). Длинные штрихи соответствуют дифракционным отражениям неупорядоченного кубического (пр. гр. $F m \overline{3} m$ ) оксигидрида титана $\mathrm{TiO}_{0.72} \mathrm{H}_{0.30}$ со структурой $B 1$. В нижней части рисунков $(a)$ и $(b)$ показаны разности $\left(I_{\mathrm{obs}}-I_{\text {calc }}\right)$ между экспериментальной и расчетной дифрактограммами оксигидрида титана $\mathrm{TiO}_{0.72} \mathrm{H}_{0.30}$.

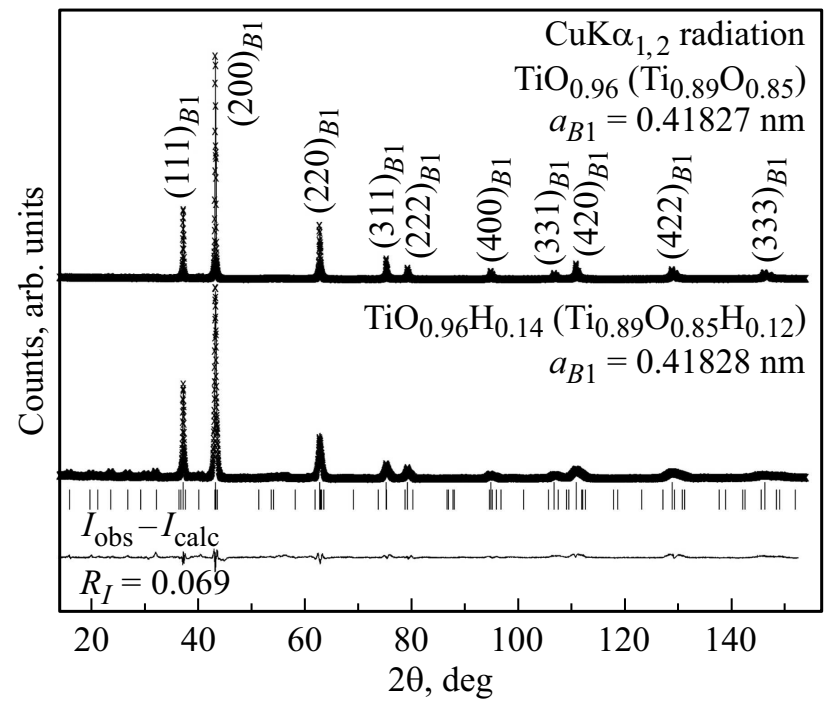

Рис. 3. Рентгенограммы исходного неупорядоченного кубического монооксида титана $\mathrm{TiO}_{0.96}\left(\mathrm{Ti}_{0.89} \mathrm{O}_{0.85}\right)$ и оксигидрида титана $\mathrm{TiO}_{0.96} \mathrm{H}_{0.14}\left(\mathrm{Ti}_{0.89} \mathrm{O}_{0.85} \mathrm{H}_{0.12}\right)(\times-$ эксперимент, сплошная линия - расчет). Исходный монооксид $\mathrm{TiO}_{0.96}$ содержит только неупорядоченную кубическую (пр. гр. $F m \overline{3} m$ ) фазу со структурой $B$. Оксигидрид титана $\mathrm{TiO}_{0.962} \mathrm{H}_{0.14}$ является двухфазным и содержит $\sim 86 \pm 2 \mathrm{wt} . \%$ неупорядоченной кубической (пр. гр. $F m \overline{3} m)$ фазы со структурой $B 1$ и $\sim 14 \pm 2$ wt.\% упорядоченной моноклинной (пр. гр. $C 2 / m$ ) фазы типа $\mathrm{Ti}_{5} \mathrm{O}_{5}$. Длинные и короткие штрихи - положения дифракционных отражений неупорядоченного кубического оксигидрида титана $\mathrm{TiO}_{0.96} \mathrm{H}_{0.14}$ со структурой $B 1$ и упорядоченной моноклинной фазы $\mathrm{Ti}_{5.33} \square_{0.67} \mathrm{O}_{5.12} \mathrm{H}_{0.74} \square_{0.14}$ типа $\mathrm{Ti}_{5} \mathrm{O}_{5}$, соответственно. $\left(I_{\mathrm{obs}}-I_{\mathrm{calc}}\right)$ - разность между экспериментальной и расчетной рентгенограммами оксигидрида титана $\mathrm{TiO}_{0.96} \mathrm{H}_{0.14}$.

После гидрирования образца $\mathrm{TiO}_{0.96}$ произошло не только образование оксигидрида $\mathrm{TiO}_{0.96} \mathrm{H}_{0.14}$, но и наряду с кубической фазой появилась вторая фаза. Анализ дифракционных данных (рис. 3) показал, что оксигидрид $\mathrm{TiO}_{0.96} \mathrm{H}_{0.14}$ содержит неупорядоченную кубическую фазу $\mathrm{Ti}_{0.89} \mathrm{O}_{0.85} \mathrm{H}_{0.12}$ в количестве $\sim 86 \pm 2$ wt.\% и упорядоченную моноклинную (пр.гр. $C 2 / m$ ) фазу типа $\mathrm{Ti}_{5} \mathrm{O}_{5}$ в количестве $\sim 14 \pm 2 \mathrm{wt} . \%$. Период решетки кубической оксигидридной фазы равен $a_{B 1}=0.41828 \mathrm{~nm}$, т. е. незначительно (в пределах ошибки измерения) увеличился вследствие внедрения водорода в вакантные позиции кислородной подрешетки. Появление моноклинной упорядоченной фазы типа $\mathrm{Ti}_{5} \mathrm{O}_{5}$ стало следствием низкотемпературного отжига образца $\mathrm{TiO}_{0.96}$, так как состав монооксида и температура гидрирования $\sim 970 \mathrm{~K}$ соответствуют области равновесного существования сверхструктуры типа $\mathrm{Ti}_{5} \mathrm{O}_{5}$.

При описании рентгенограммы (рис. 3) оксигидрида $\mathrm{TiO}_{0.96} \mathrm{H}_{0.14}$ наилучшая сходимость эксперимента и расчета достигнута при статистическом размещении всех атомов водорода в вакантных октаэдрических позициях кислородной подрешетки: в случае неупорядоченной 
Таблица 2. Моноклинная (пр.гр. $C 2 / m$ ) оксигидридная фаза $\mathrm{Ti}_{5.33} \mathrm{O}_{5.12} \mathrm{H}_{0.74}$ со структурой типа $\mathrm{Ti}_{5} \mathrm{O}_{5}\left(a_{m}=0.58488 \mathrm{~nm}\right.$, $\left.b_{m}=0.41473 \mathrm{~nm}, c_{m}=0.94562 \mathrm{~nm}, \beta=108.26^{\circ}\right)$, содержащаяся в двухфазном оксигидриде $\mathrm{TiO}_{0.96} \mathrm{H}_{0.14}$ в количестве $\sim 14 \pm 2 \mathrm{wt} . \%$

\begin{tabular}{|c|c|c|c|c|c|c|c|c|}
\hline \multirow{2}{*}{ Атом } & \multirow{2}{*}{$\begin{array}{c}\text { Позиция } \\
\text { и кратность }\end{array}$} & \multicolumn{3}{|c|}{$\begin{array}{l}\text { Атомные координаты } \\
\text { в упорядоченной фазе }\end{array}$} & \multicolumn{3}{|c|}{$\begin{array}{c}\text { Атомные координаты } \\
\text { в базисной структуре } B 1\end{array}$} & \multirow{2}{*}{$\begin{array}{c}\text { Значения функций распределения } \\
n_{\mathrm{Ti}}\left(x_{\mathrm{I}} y_{\mathrm{I}}, z_{\mathrm{I}}\right), n_{\mathrm{O}}\left(x_{\mathrm{I}}, y_{\mathrm{I}}, z_{\mathrm{I}}\right) \text { и } n_{\mathrm{H}}\left(x_{\mathrm{I}}, y_{\mathrm{I}}, z_{\mathrm{I}}\right)\end{array}$} \\
\hline & & $x / a_{m}$ & $y / b_{m}$ & $z / c_{m}$ & $x_{\mathrm{I}}$ & $y_{\mathrm{I}}$ & $z_{\mathrm{I}}$ & \\
\hline Ti 1 (вакансия) & $2(a)$ & 0 & 0 & 0 & 0 & 0 & 0 & $n_{1(\mathrm{Ti})}=x-\eta_{10}^{\mathrm{Ti}} / 6-\eta_{4}^{\mathrm{Ti}} / 3-\eta_{1}^{\mathrm{Ti}} / 3 \approx 0.328$ \\
\hline Ti 2 & $2(d)$ & $1 / 2$ & $1 / 2$ & $1 / 2$ & 1 & $1 / 2$ & $1 / 2$ & $n_{2(\mathrm{Ti})}=x+\eta_{10}^{\mathrm{Ti}} / 6-\eta_{4}^{\mathrm{Ti}} / 3+\eta_{1}^{\mathrm{Ti}} / 3 \approx 1$ \\
\hline Ti 3 & $4(i)$ & 0.1654 & 0 & 0.3425 & 0.5079 & 0 & 0.5196 & $n_{3(\mathrm{Ti})}=x+\eta_{10}^{\mathrm{Ti}} / 6+\eta_{4}^{\mathrm{Ti}} / 6-\eta_{1}^{\mathrm{Ti}} / 6 \approx 1$ \\
\hline $\mathrm{Ti} 4$ & $4(i)$ & 0.3218 & 0 & 0.6652 & 0.9870 & 0 & 1.0086 & $n_{4(\mathrm{Ti})}=x-\eta_{10}^{\mathrm{Ti}} / 6+\eta_{4}^{\mathrm{Ti}} / 6+\eta_{1}^{\mathrm{Ti}} / 6 \approx 1$ \\
\hline О 1 (вакансия) & $2(c)$ & $1 / 2$ & 0 & $1 / 2$ & 1 & 0 & $1 / 2$ & $n_{1(\mathrm{O})}=z-\eta_{10}^{\mathrm{O}} / 6-\eta_{4}^{\mathrm{O}} / 3-\eta_{1}^{\mathrm{O}} / 3 \approx 0.124$ \\
\hline $\mathrm{O} 2$ & $2(b)$ & 0 & $1 / 2$ & 0 & 0 & $1 / 2$ & 0 & $n_{2(\mathrm{O})}=z+\eta_{10}^{\mathrm{O}} / 6-\eta_{4}^{\mathrm{O}} / 3+\eta_{1}^{\mathrm{O}} / 3 \approx 1$ \\
\hline O 3 & $4(i)$ & 0.3364 & 0 & 0.1681 & 0.5045 & 0 & 0.0002 & $n_{3(\mathrm{O})}=z+\eta_{10}^{\mathrm{O}} / 6+\eta_{4}^{\mathrm{O}} / 6-\eta_{1}^{\mathrm{O}} / 6 \approx 1$ \\
\hline O 4 & $4(i)$ & 0.1668 & 0 & 0.8322 & 0.0010 & 0 & 0.4976 & $n_{4(\mathrm{O})}=z-\eta_{10}^{\mathrm{O}} / 6+\eta_{4}^{\mathrm{O}} / 6+\eta_{1}^{\mathrm{O}} / 6 \approx 1$ \\
\hline Н 1 (вакансия) & $2(c)$ & $1 / 2$ & 0 & $1 / 2$ & 1 & 0 & $1 / 2$ & $n_{1(\mathrm{H})}=p x+\eta_{10}^{\mathrm{H}} / 6+\eta_{4}^{\mathrm{H}} / 3+\eta_{1}^{\mathrm{H}} / 3 \approx 0.744$ \\
\hline $\mathrm{H} 2$ & $2(b)$ & 0 & $1 / 2$ & 0 & 0 & $1 / 2$ & 0 & $n_{2(\mathrm{H})}=p x-\eta_{10}^{\mathrm{H}} / 6+\eta_{4}^{\mathrm{H}} / 3-\eta_{1}^{\mathrm{H}} / 3 \approx 0$ \\
\hline H 3 & $4(i)$ & 0.3364 & 0 & 0.1681 & 0.5045 & 0 & 0.0002 & $n_{3(\mathrm{H})}=p x-\eta_{10}^{\mathrm{H}} / 6-\eta_{4}^{\mathrm{H}} / 6+\eta_{1}^{\mathrm{H}} / 6 \approx 0$ \\
\hline H 4 & $4(i)$ & 0.1668 & 0 & 0.8322 & 0.0010 & 0 & 0.4976 & $n_{4(\mathrm{H})}=p x+\eta_{10}^{\mathrm{H}} / 6-\eta_{4}^{\mathrm{H}} / 6-\eta_{1}^{\mathrm{H}} / 6 \approx 0$ \\
\hline
\end{tabular}

кубической фазы это позиции $4(b)$, а в моноклинной фазе это позиции $2(c)$.

Проведенный количественный анализ структурных рентгеновских данных показал, что присутствующая в оксигидриде $\mathrm{TiO}_{0.96} \mathrm{H}_{0.14}$ вторая фаза (упорядоченный моноклинный оксигидрид типа $\mathrm{Ti}_{5} \mathrm{O}_{5}$ ) имеет состав $\mathrm{Ti}_{5.33} \square_{0.67} \mathrm{O}_{5.12} \mathrm{H}_{0.74} \square_{0.14}\left(\mathrm{Ti}_{0.89} \mathrm{O}_{0.85} \mathrm{H}_{0.12}\right)$ (табл. 2). Согласно [17] и выполненному анализу, упорядоченный моноклинный оксигидрид типа $\mathrm{Ti}_{5} \mathrm{O}_{5}$ образуется по каналу фазового перехода, включающему один луч лифшицевской звезды $\left\{\mathbf{k}_{10}\right\}$ и по два луча нелифшицевских звезд $\left\{\mathbf{k}_{4}\right\}$ и $\left\{\mathbf{k}_{1}\right\}$ (нумерация звезд $\left\{\mathbf{k}_{s}\right\}$ волновых векторов дана в соответствии с $[1,22])$.

Выполненный расчет показал, что функции распределения атомов титана, кислорода и водорода в наблюдаемой моноклинной сверхструктуре типа $\mathrm{Ti}_{5} \mathrm{O}_{5}$ оксигидрида $\mathrm{Ti}_{0.89} \mathrm{O}_{0.85} \mathrm{H}_{0.12}$ с $x=0.89, z=0.85$ и $p x=0.12$ имеют вид

$$
\begin{aligned}
& n_{\mathrm{Ti}}\left(x_{\mathrm{I}}, y_{\mathrm{I}}, z_{\mathrm{I}}\right)=x-\left(\eta_{10}^{\mathrm{Ti}} / 6\right) \cos 2 \pi z_{\mathrm{I}} \\
& -\left(\eta_{4}^{\mathrm{Ti}} / 3\right) \cos \left[4 \pi\left(x_{\mathrm{I}}+z_{\mathrm{I}}\right) / 3\right]-\left(\eta_{1}^{\mathrm{Ti}} / 3\right) \cos \left[2 \pi\left(2 x_{\mathrm{I}}-z_{\mathrm{I}}\right) / 3\right], \\
& n_{\mathrm{O}}\left(x_{\mathrm{I}}, y_{\mathrm{I}}, z_{\mathrm{I}}\right)=z+\left(\eta_{10}^{\mathrm{O}} / 6\right) \cos 2 \pi z_{\mathrm{I}} \\
& -\left(\eta_{4}^{\mathrm{O}} / 3\right) \cos \left[4 \pi\left(x_{\mathrm{I}}+z_{\mathrm{I}}\right) / 3\right]+\left(\eta_{1}^{\mathrm{O}} / 3\right) \cos \left[2 \pi\left(2 x_{\mathrm{I}}-z_{\mathrm{I}}\right) / 3\right], \\
& n_{\mathrm{H}}\left(x_{\mathrm{I}}, y_{\mathrm{I}}, z_{\mathrm{I}}\right)=p x-\left(\eta_{10}^{\mathrm{H}} / 6\right) \cos 2 \pi z_{\mathrm{I}} \\
& +\left(\eta_{4}^{\mathrm{H}} / 3\right) \cos \left[4 \pi\left(x_{\mathrm{I}}+z_{\mathrm{I}}\right) / 3\right]-\left(\eta_{1}^{\mathrm{H}} / 3\right) \cos \left[2 \pi\left(2 x_{\mathrm{I}}-z_{\mathrm{I}}\right) / 3\right] .
\end{aligned}
$$

В функциях (8), (9) и (10) $n\left(x_{\mathrm{I}}, y_{\mathrm{I}}, z_{\mathrm{I}}\right)$ - вероятность нахождения соответствующего атома в узле $\mathbf{r}$ упорядочивающейся подрешетки с базисными кубическими координатами $x_{\mathrm{I}}, y_{\mathrm{I}}, z_{\mathrm{I}} ; \eta_{s}\left(\eta_{10}, \eta_{4}, \eta_{1}\right)$ - параметры дальнего порядка, соответствующие звездам $\left\{\mathbf{k}_{10}\right\},\left\{\mathbf{k}_{4}\right\}$ и $\left\{\mathbf{k}_{1}\right\}$.

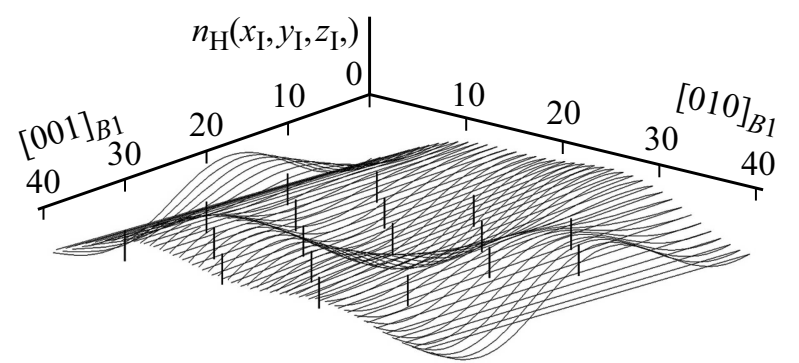

Рис. 4. Функция распределения $n_{\mathrm{H}}\left(x_{\mathrm{I}}, y_{\mathrm{I}}, z_{\mathrm{I}}\right)(10)$ атомов водорода в плоскости $\left(\begin{array}{lll}1 & -1 & 1\end{array}\right)_{B 1}$ мононоклинного (пр. гр. $\left.C 2 / m\right)$ оксигидрида титана $\mathrm{Ti}_{5.33} \mathrm{O}_{5.12} \mathrm{H}_{0.74}$. Положение узлов водородной подрешетки, с вероятностью $\sim 0.74$ занятых атомами $\mathrm{H}$, показано вертикальными линиями.

В [17] показано, что в сверхструктуре типа $\mathrm{Ti}_{5} \mathrm{O}_{5}$ параметры $\eta_{s}$, описывающие конкретную подрешетку, равны, но каждой из подрешеток соответствует своя величина $\eta_{s}$. Для перехода от моноклинных координат к кубическим используются соотношения $x_{\mathrm{I}}=x_{m}+z_{m}, y_{\mathrm{I}}=y_{m}$ и $z_{\mathrm{I}}=-x_{m}+2 z_{m}$, Согласно расчету, в приближении равных параметров дальнего порядка для подрешеток титана, кислорода и водорода $\eta_{10}^{\mathrm{Ti}}=\eta_{4}^{\mathrm{Ti}}=\eta_{1}^{\mathrm{Ti}} \approx 0.674$, $\eta_{10}^{\mathrm{O}}=\eta_{4}^{\mathrm{O}}=\eta_{1}^{\mathrm{O}} \approx 0.871$ и $\eta_{10}^{\mathrm{H}}=\eta_{4}^{\mathrm{H}}=\eta_{1}^{\mathrm{H}} \approx 0.749$.

На рис. 4 как пример показана функция распределения $n_{\mathrm{H}}\left(x_{\mathrm{I}}, y_{\mathrm{I}}, z_{\mathrm{I}}\right)(10)$ атомов водорода в плоскости $(1-11)$ моноклинного (пр. гр. $C 2 / m$ ) оксигидридеа титана $\mathrm{Ti}_{5.33} \mathrm{O}_{5.12} \mathrm{H}_{0.74}$ и положение узлов водородной подрешетки, с вероятностью $\sim 0.74$ занятых атомами $\mathrm{H}$.

\section{3. Заключение}

Изучение структуры оксигидридов титана $\mathrm{TiO}_{y} \mathrm{H}_{p}$ показало, что оксигидриды сохраняют кристаллическую 
решетку типа $B 1$ исходных кубических монооксидов и содержат структурные вакансии в металлической и кислородной подрешетках. В кубических (пр.гр. $F m \overline{3} m$ ) оксигидридах внедренные атомы водорода занимают вакантные октаэдрические позиции $4(b)$ кислородной подрешетки, размешения водорода в тетраэдрических позициях $8(c)$ не наблюдается. При образовании моноклинной (пр.гр. $C 2 / m$ ) оксигидридной упорядоченной фазы типа $\mathrm{Ti}_{5} \mathrm{O}_{5}$ атомы $\mathrm{H}$ размещаются только в вакантных октаэдрических позициях 2(c) кислородной подрешетки, т.е. в окружении шести узлов подрешетки титана.

\section{Благодарности}

Авторы благодарят А.В. Скрипова (ИФМ УрО РАН) за гидрирование монооксидов титана, Н. Wu и T.J. Udovic (NIST Center for Neutron Research, Gaithersburg, USA) за помощь в нейтронографических измерениях.

\section{Финансирование работы}

Работа выполнена по государственному заданию № 0397-2019-0001 в Институте химии твердого тела Уральского отделения Российской академии наук.

\section{Конфликт интересов}

Авторы заявляют об отсутствии конфликта интересов.

\section{Список литературы}

[1] A.I. Gusev, A.A. Rempel, A.J. Magerl. Disorder and Order in Strongly Nonstoichiometric Compounds: Transition Metal Carbides, Nitrides and Oxides, Springer: Heidelberg (2001). $608 \mathrm{p}$.

[2] А.А. Валеева, А.А. Ремпель, С.В. Ремпель, С.И. Садовников, А.И. Гусев. Успехи химии 90, 5, 601 (2021).

[3] H. Goretzki. Phys. Status Solidi 20, 2, K141 (1967).

[4] K. Yvon, H. Nowotny, R. Kieffer. Monatsh. Chem. 98, 12, 2164 (1967).

[5] В.Т. Эм, И. Каримов, В.Ф. Петрунин, И.С. Латергаус, М.М. Антонова, И.И. Тимофеева, В.Я. Науменко. ФММ 39, 6, 1286 (1975).

[6] S. Rundqvist, R. Tellgren, Y. Andersson. J. Less-Common Met. 101, 145 (1984).

[7] G. Renaudin, K. Yvon, S.K. Dolukhanyan, N.N. Aghajanyan, V.S. Shekhtman. J. Alloys Comp. 356-357, 120 (2003).

[8] И.С. Латергаус, И. Каримов, В.С. Пресман, В.Т. Эм. Изв. АН СССР. Неорган. материалы 23, 9, 1471 (1987).

[9] A.V. Skripov, H. Wu, T.J. Udovic, Q. Huang, R. Hempelmann, A.V. Soloninin, A.A. Rempel, A.I. Gusev. J. Alloys Comp. 478, $1-2,68$ (2009).

[10] A.V. Skripov, T.J. Udovic, J.C. Cook, R. Hempelmann, A.A. Rempel, A.I. Gusev. J. Phys.: Condens. Matter 21, 17, 175410 (2009).

[11] G.W. Samsonow, W.W. Morosow. Monatsh. Chem. 102, 6, 1667 (1971).
[12] И.Е. Павлов, С.И. Алямовский, Ю.Г. Зайнулин, Г.П. Швейкин. Порошк. металлургия 10, 34 (1975).

[13] A. San-Martin, F.D. Manchester. Bull. Alloy Phase Diagrams 8, 1, 30 (1987).

[14] V.F. Sear. Neutron News 3, 3, 26 (1992).

[15] Ю.З. Нозик, Р.П. Озеров, К. Хенниг. Структурная нейтронография, Атомиздат, М. (1979).

[16] А.А. Валеева, А.А. Ремпель, А.И. Гусев. Неорган. материалы 37, 6, 716 (2001).

[17] А.А. Валеева, А.А. Ремпель, А.И. Гусев. Письма в ЖЭТФ 71, 11, 675 (2000).

[18] A.A. Rempel, W. van Renterghem, A.A. Valeeva, M. Verwerft, S. van den Berghe. Sci. Reports 7, 10769 (2017).

[19] J.K. Stalick, E. Prince, A. Santoro, I.G. Schroder, J.J. Rush. In: Neutron Scattering in Materials Science II / Eds. D.A. Neumann, T.P. Russel, B.J. Wuensch. NIST, Materials Research Society: Pittsburgh, PA, USA (1995). P. 101-106.

[20] X'Pert HighScore Plus. Version 2.2e (2.2.5). (C) 2009 PANalytical B.V. Almedo, the Netherlands.

[21] A.V. Skripov, A.V. Soloninin, A.A. Valeeva, A.I. Gusev, A.A. Rempel, H. Wu, T.J. Udovic. J. Alloys Comp. 887, 161353 (2021).

[22] О.В. Ковалев. Неприводимые и индуцированные представления и копредставления федоровских групп. Наука, M.(1986). 368 c.

Редактор Д.В. Жуманов 